\title{
Disease prestige and the hierarchy of suffering
}

\author{
Suffering should not be hierarchical, and care should not be predicated on the \\ prestige that a disease attracts
}

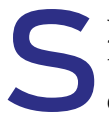

ymptoms may herald illness, but it is the diagnosis that announces the presence of disease. While the experience of illness is subjective, disease is authorised by a health professional through diagnosis (Box 1). ${ }^{1}$ A good diagnosis explains pathology, suggests prognosis, enables access to services, grounds evidencebased therapies and provides an explanation that makes sense of a patient's suffering. Beyond this, a diagnosis justifies sickness, providing the patient with a rationale for their disabilities - for friends, family, employees, but most importantly for patients themselves. ${ }^{2}$ To be left without a diagnosis is to be left without a story, with no way of making sense of suffering or communicating distress to others. Diagnosis, then, is often a relief, even when the diagnosis suggests a bleak future ("thank goodness, I knew there was something wrong"). ${ }^{3}$

However, not all diagnoses are equal in the eyes of the world in which we live (Box 2). In the lottery of illness, care can vary across the diagnostic spectrum, depending on the profile of the disease. The idea of disease hierarchy was discussed as early as 1943, when prestige was seen to be "based on the extent to which symptoms can - or cannot - be readily localized". ${ }^{4}$ Album and Westin suggested that "diseases and specialities associated with technologically sophisticated, immediate and invasive procedures in vital organs located in the upper parts of the body are given high prestige scores, especially where the typical patient is young or middle-aged ... low prestige scores are given to diseases and specialities associated with chronic conditions located in the lower parts of the body or having no specific bodily location, with less visible treatment procedures, and with elderly patients."

High prestige illnesses, such as breast cancer, many childhood cancers and heart disease, are well understood by the population, and clearly recognised as illnesses that deserve support and care. Many high prestige illnesses are common, and attract significant philanthropic support through well organised, well funded campaigns. (eg, the pinkification of breast cancer). ${ }^{6}$ Celebrity patronage facilitates fundraising, supports awareness campaigns and suggests topical articles in the popular press, although it can also alienate patients who do not fit the marketing hype (eg, men with breast cancer). ${ }^{7}$ High prestige diseases have support groups that share narratives of suffering and recovery, provide consumer

Louise Stone

Australian National University, Canberra, ACT.

louise.stone@ anu.edu.au expertise to assist patients and their carers to navigate the complexity of health care, and create opportunities to understand, participate in and fund research. For sufferers of cancer, fear and uncertainty may be eased by the presence of cancer care centres. Philanthropy and public funding enable these centres to offer everything from accessible parking, to wig and beauty services, to comprehensive clinical care.

\begin{abstract}
1 Modes of unhealth
Disease: a pathological process that includes deviation from a biological norm

"Diseases are valued as central facts in the medical view."

Illness: a subjective experience of unhealth which is interior to the person of the patient

Illness may occur without disease, or before disease is diagnosed (eg, prodromes). Medically unexplained illness is uncomfortable for the clinician and distressing for the patient: "The patient can offer the doctor nothing to satisfy his senses - he can only bring messages of pain to the doctor, from an underworld of experience shut off for ever from the clinical gaze."

\section{Sickness: an external and public mode of unhealth}

Sickness is a social role that is negotiated between the person and the society which supports them. The security of the sick role depends on "the possession of that much treasured gift, the disease... But even the possession of disease does not guarantee equity in sickness. Those with a chronic disease are much less secure than those with an acute one; those with a psychiatric disease than those with a surgical one... Best is an acute physical disease in a young man quickly determined by recovery or death - either will do, both are equally regarded."
\end{abstract}

However, there are diseases that are less well supported. Some diseases are less easy to understand or communicate (eg, metabolic disorders) or may make people in the community uncomfortable (eg, anal cancer). Other diseases are seen as less worthy, and community members may perceive that illness is the person's own fault (eg, alcoholic liver disease). Sufferers of lung cancer, even those who have been non-smokers, describe distressing interactions with people in the community, who do not feel they deserve care because they are assumed to be smokers who have caused their own suffering. ${ }^{8}$ These diseases are less well supported - not just clinically, where there is less philanthropic support to stretch the public dollar, but also in research, where there

\section{Disease prestige and stigma}

Disease prestige is the collective perception of a disease's "worthiness"; the degree to which the sufferer "deserves" care and support. As a social construct, it changes over time and between communities. While health is obviously more desirable than disease, a disease's prestige reflects the community's commitment to care, support and economic investment.

Stigma is the personal experience of feeling diminished and devalued because of negative social beliefs about the patient and his or her disease.

Stigma and disease prestige have a complex relationship: some diseases (eg, depression, HIV/AIDS) attract considerable social support despite the stigma experienced by sufferers, whereas others (eg, schizophrenia, urinary incontinence) do not. 
are fewer community sponsored grants. ${ }^{9}$ Interestingly, the relationship between stigma and self-determination is not always clear. For instance, there are lifestyle risk factors for both lung cancer and cardiovascular disease, but heart disease is high prestige and lung cancer is not.

Highly stigmatising diseases are even less supported. Patients with embarrassing diseases (eg, inflammatory bowel disease, urinary incontinence) may keep their disease secret due to shame. Patients with mental illnesses often avoid disclosing their illness because of active discrimination in the workplace, at home or by institutions such as insurance companies. ${ }^{10}$ This has a profound impact on help seeking and the ability of health care teams to provide appropriate care and support. However, the relationship between stigma and prestige is not always clear either; depression has become a high prestige disease in recent times, despite the ongoing stigma surrounding mental illness. Other diseases with intense community support (eg, HIV / AIDS and breast cancer) were once hidden and poorly supported.

Diseases with the lowest prestige attract discrimination in the health care sector as well as in the community. Many are ill defined, and exist as syndromes without a common understanding of their nature or pathology. This is particularly true in psychiatry where taxonomy is constantly changing to adapt to our evolving understanding what psychiatric illness actually is. Psychiatric disorders still sit uneasily across foundations in neurology, biology, psychology, sociology and philosophy, among other fields of human concern. The sad irony is that many patients with psychiatric illness are survivors of childhood abuse; their needs were not acknowledged and met in childhood and, in adulthood, they have acquired a disease label that precipitates similar invalidation in the health care system. This retraumatising experience is inappropriate, unhelpful and often cruel. Some of these patients describe how the diagnosis follows them around like a criminal record, impeding their ability to obtain appropriate care, even for unrelated physical illness. ${ }^{11}$ In the community, many of these patients experience distrust and profound isolation - they "cannot face engaging with a process that invalidates their pain". ${ }^{12}$

Finally, there are the unnamed conditions where there is no diagnosis, the so-called medically unexplained illnesses. With no disease name, these patients struggle to access care, and have no language or concepts to help them make sense of their suffering. They are the "heartsink" patients in medicine and "hypochondriacs" in the community: invalidating, unhelpful and judgemental terms that blame the victims for their own distress. Many patients with medically unexplained symptoms attach themselves to online communities that invest enormous energy in establishing a disease name that will give them the validation they need, in health care and in the community. ${ }^{13}$ In essence, many of these contested illnesses "behave like an infectious disease with a sociocultural vector", 14 changing their shape and character over time as illness labels come and go. Contested illness labels may provide patients with a sense of community, but they often rest on a cluster of non-specific symptoms like fatigue, non-specific pain or weakness, and therefore attract a diverse group of sufferers with little in common. Nevertheless, online communities create very powerful spaces for effective medicalisation through peer-to-peer networks. Patients who attach themselves to contested disease labels describe their health care as a battleground, where they constantly fight for recognition and validation for their suffering. ${ }^{15}$ Some of the communication in these groups undoubtedly offers participants a chance to share and rehearse strategies to obtain their preferred diagnoses, treatments, referrals and certification.

The lower the disease prestige, the less support is available to the patient in the community. However, there is also a hierarchy of evidence that makes it difficult for many low prestige diseases to access research funding, generate an evidence base or argue for specific interventions. The gold standard in this hierarchy is the systematic review or meta-analysis of quality randomised controlled trials. However, randomised controlled trials have their limitations. They are ideal for easily defined, high prevalence illness with specific, well defined interventions, but good quality evidence may be difficult to generate for low prevalence disorders, diseases that are ill defined and interventions that are difficult to standardise. Similarly, evidence is often lacking for

3 Practical strategies to support a patient with low prestige and medically unexplained illness ${ }^{13}$

Validation

- Acknowledge that the symptoms are real and distressing

- Acknowledge that medicine has limits and the uncertainty is frustrating

- Recognise the effects of community stigma and acknowledge that this is painful

\section{Explanation}

- Consider and record physical, psychiatric and psychosocial diagnoses and symptoms

- Give careful explanations about what is known about the disease, and share any resources available

\section{Coordination of care and advocacy}

- Coordinate care to avoid duplication of investigations and exacerbation of iatrogenic harm

- Advocate for access to appropriate services and support

- Continue to measure function and quality of life as an index of illness severity

Illness management

- Offer symptom relief and practical support to address disability (eg, home help, workplace assessment)

- Encourage physical therapies (eg, massage, physiotherapy, hydrotherapy)

- Manage comorbidities as effectively as possible

- Encourage psychological care to address the impact of illness and underlying issues that may exacerbate symptoms

- Discuss healthy lifestyle goals

- Minimise harm by avoiding unhelpful investigations and treatments

- Check for new diagnoses when the illnesses changes significantly (eg, the emergence of a new symptom) or during a yearly health check

\section{Empathy}

- Manage the therapeutic relationship carefully and seek support if it becomes unhelpful 
populations that are harder to access (eg, certain cultural groups) or groups that pose particular ethical challenges (eg, very old people, patients with intellectual disability). In primary care, this often means that lower prestige diseases have less evidence to guide care.

As health care practitioners, our goal is not to classify diseases, but to manage the impact of illness on the individual to reduce suffering. ${ }^{16}$ This is the difference between science and medicine and is the ethical task of our profession. Regardless of where on the hierarchy our patient's disease resides, we need to mobilise the resources that do exist, advocate for resources that are not available and support patients who suffer with few resources at all. This includes working with patients whose condition lacks evidence to guide clinical decision making.

It is critical that the medical community is able to validate suffering, make sense of it and manage it, no matter how little prestige the illness attracts. The lower the disease prestige, the greater the need for doctors to fill the void in validation and support (Box 3). It is also critical that as a profession, we consider our disease-bounded models of health care delivery and research. We have strategies for understanding burden of disease, but less developed strategies for understanding burden of illness. We need to consider issues of justice and equity, not only across populations but also between diseases, and we need to create overt clinical, educational and research priorities that recognise the complexities of funding the breadth of illness that occurs in the community. Suffering should not be hierarchical, and care should not be predicated on the prestige that a disease attracts.

Competing interests: No relevant disclosures.

Provenance: Not commissioned; externally peer reviewed.

(c) 2018 AMPCo Pty Ltd. Produced with Elsevier B.V. All rights reserved.

References are available online at www.mja.com.au. 
1 Marinker M. Why make people patients? J Med Ethics 1975; 1: 81-84.

2 Nettleton S. 'I just want permission to be ill': towards a sociology of medically unexplained symptoms. Social Sci Med 2006; 62: 1167-1178.

3 Nettleton S, O'Malley L, Watt I, Duffey P. Enigmatic illness: narratives of patients who live with medically unexplained symptoms. Social Theory Health 2004; 2: 47-66.

4 Canguilhem $\mathrm{G}$. On the normal and the pathological. New York: Zone Books, 1991.

5 Album D, Westin S. Do diseases have a prestige hierarchy? A survey among physicians and medical students. Social Sci Med 2008; 66: 182-188.

6 King S. Pink Ribbons Inc: breast cancer activism and the politics of philanthropy. Int J Qual Stud Educ 2004; 17: 473-492.

7 Chapman S, McLeod K, Wakefield M, Holding S. Impact of news of celebrity illness on breast cancer screening: Kylie Minogue's breast cancer diagnosis. Med J Aust 2005; 183: 247-250. https://www.mja.com.au/journal/ 2005/183/5/impact-news-celebrity-illness-breast-cancer-screening-kylieminogues-breast

8 Chapple A, Ziebland S, McPherson A. Stigma, shame, and blame experienced by patients with lung cancer: qualitative study. BMJ 2004; 328: 1470.
9 Viergever RF. The mismatch between the health research and development (R\&D) that is needed and the R\&D that is undertaken: an overview of the problem, the causes, and solutions. Glob Health Action 2013; 6; https://doi. org/10.3402/gha.v6i0.22450.

10 Clement S, Schauman O, Graham T, et al. What is the impact of mental health-related stigma on help-seeking? A systematic review of quantitative and qualitative studies. Psychol Med 2015; 45: 11-27.

11 Stone L. Blame, shame and hopelessness: medically unexplained symptoms and the 'heartsink' experience. Aust Fam Physician 2014; 43: 191.

12 McGowan L, Luker K, Creed F, Chew-Graham CA. 'How do you explain a pain that can't be seen?': the narratives of women with chronic pelvic pain and their disengagement with the diagnostic cycle. Br J Health Psychol 2007; 12: 261-274.

13 Stone L. Managing medically unexplained illness in general practice. Aust Fam Physician 2015; 44: 624.

14 Ryder AG, Chentsova-Dutton YE. Depression in cultural context: "Chinese somatization," revisited. Psychiatr Clin North Am 2012; 35: 15-36.

15 Dumit J. Illnesses you have to fight to get: facts as forces in uncertain, emergent illnesses. Social Sci Med 2006; 62: 577-590.

16 Sadler JZ. Values and psychiatric diagnosis. Oxford: Oxford University Press, 2005. 